Running head: The selection balance

\title{
The selection balance: contrasting value, proximity and priming in a multitarget foraging task
}

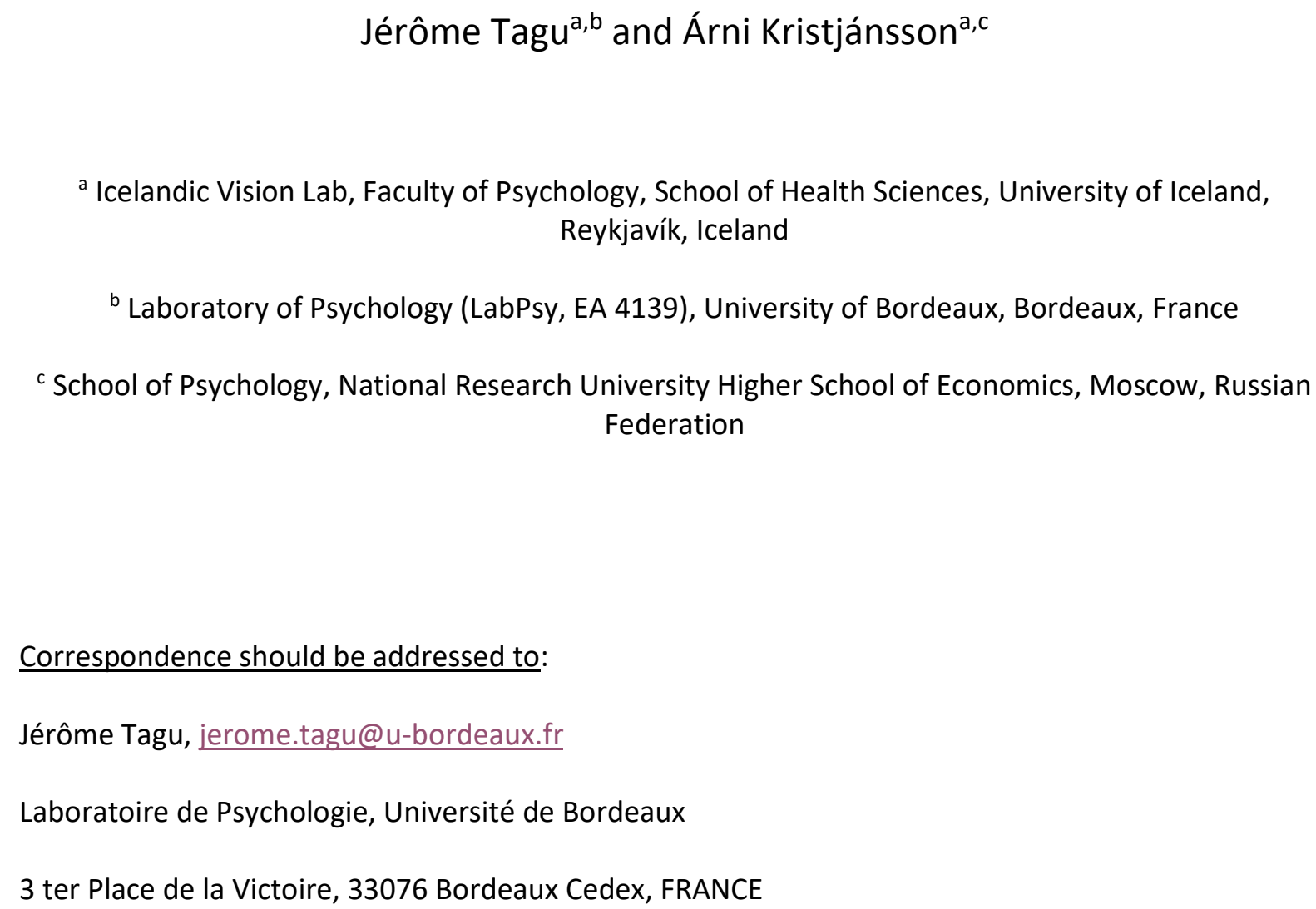

Correspondence should be addressed to:

Jérôme Tagu, jerome.tagu@u-bordeaux.fr

Laboratoire de Psychologie, Université de Bordeaux

3 ter Place de la Victoire, 33076 Bordeaux Cedex, FRANCE 
Abstract

A critical question in visual foraging concerns the mechanisms driving the next target selection. Observers first identify a set of candidate targets, and then select the best option among these candidates. Recent evidence suggests that target selection relies on internal biases towards proximity (nearest target from the last selection), priming (target from the same category as the last selection) and value (target associated with high value). Here, we tested the role of eye movements in target selection, and notably whether disabling eye movements during target selection could affect search strategy. We asked observers to perform four foraging tasks differing by selection modality and target value. During gaze foraging, participants had to accurately fixate the targets to select them and could not anticipate the next selection with their eyes, while during mouse foraging they selected the targets with mouse clicks and were free to move their eyes. We moreover manipulated both target value and proximity. Our results revealed notable individual differences in search strategy, confirming the existence of internal biases towards value, proximity and priming. Critically, there were no differences in search strategy between mouse and gaze foraging, suggesting that disabling eye movements during target selection did not affect foraging behaviour. These results importantly suggest that overt orienting is not necessary for target selection. This study provides fundamental information for theoretical conceptions of attentional selection, and emphasizes the importance of covert attention for target selection during visual foraging. 


\section{Introduction}

While a large amount of research has been devoted to how humans visually search for targets in their environment, this research has for the most part involved search for one unique target among several distractors (Hulleman \& Olivers, 2017; Treisman \& Gelade, 1980; Wolfe, 2010; Wolfe \& Horowitz, 2017). Recently, multi-target displays have been used to assess orienting in the visual field (e.g., Cain et al., 2012; Fougnie et al., 2015; Hills et al., 2013; Wolfe, 2013; Wolfe et al., 2016), and the results have raised a number of challenges for theories of visual attention (Á. Kristjánsson et al., 2014; T. Kristjánsson et al., 2018, 2020; Tagu \& Kristjánsson, 2020), calling for further characterization of visual foraging under different conditions.

One critical question in visual foraging concerns the factors driving the next target selection. This is likely to require two steps: identifying a set of candidate targets for the next selection, and then selecting the best option among these candidates (Wolfe et al., 2018, 2019). In a recent study, Wolfe et al. (2018) investigated which factors determine the second step, the selection of the best candidate, and examined whether the value assigned to different target types and the prevalence of target types could affect foraging behaviour. This is an important issue as our interactions with the world often involve targets of different value (e.g., coins in a purse, our favourite candies in a full bowl). In their foraging task, each stimulus was associated with a different number of points and participants were asked to earn a pre-specified number of points as quickly as possible. The prevalence of the targets was manipulated as well, so that in one of the conditions the most valuable targets were the rarest in the display. Observers could move to another stimulus display whenever they wanted, and the authors were interested in the time at which observers chose to move to the next display and in the identity of the targets that they had left behind. Wolfe et al. (2018) showed that when the most valuable targets were fewer on screen, some observers chose to only pursue rare but valuable targets and to move to the next display as soon as these targets had been erased, while others chose to continue foraging for other more common but less valuable targets before moving to the next display. But in 
other conditions where all targets had the same value, observers either prioritized targets from the most prevalent category in the display or the targets closest to the previous selection. Note that the influence of target prevalence on search performance has also been found in single-target visual search (Wolfe \& Van Wert, 2010). These results led Wolfe et al. (2018) to the conclusion that three main forces determine the next target selection: target value, spatial proximity to the previous selection, and priming of the previous target features. In other words, these three forces compete with one another, and depending on the conditions and on the individual's internal biases, the "winner" of the competition is the target selected next.

Recent evidence has identified several factors that could bias the competition in favour of value, proximity or priming. For example, Á. Kristjánsson et al. (2014) showed that during feature-based foraging, observers tend to locate the nearest target from the previous selection and frequently switch between target types for consecutive selections, while during conjunction foraging, they tend to focus on a single target type until the entire category is exhausted. In other words, this study revealed that task difficulty (or target crypticity) is an important factor for determining the next target selection, easy feature-based foraging favouring proximity to the previous target and difficult conjunction-based foraging favouring the primed features of the previous target. Later studies have identified other factors that play a role in determining the next target selection. Selection modality seems to be an important factor, as foraging with a computer mouse or with an infrared hand tracker made participants more likely to switch between target categories, favouring proximity, compared with foraging with fingers on a touchscreen (Thornton et al., 2019) or with eye gaze on a gaze-contingent display (Tagu \& Kristjánsson, 2020) where participants tended to select items from the same category as on previous selections, favouring priming. Importantly, these different selection modalities are associated with different temporal dynamics, that may have contributed to the observed results. For example, the inter-target time, which corresponds to the time that has elapsed between two 
successive target selections, is much lower during finger foraging (about $360 \mathrm{~ms}^{1}$ in Á. Kristjánsson et al., 2014) than during mouse foraging (about $580 \mathrm{~ms}^{1}$ in Tagu \& Kristjánsson, 2020). Increased time between target selections may allow participants to switch more easily between target categories, prioritising proximity over priming. Thornton et al. (2020) directly tested this potential influence of time constraints upon foraging strategy by asking participants to synchronise their foraging speed with an auditory metronome signal and by manipulating the tempo of this signal. Their results clearly showed that with slow tempo, participants located the nearest targets and prioritised proximity, but that with increasing tempo, they were more likely to select items from the same category as previous selections and to prioritise priming. Importantly, this was found both during feature-based and conjunction-based foraging, suggesting that task difficulty and time constraints are two different factors that play a role in determining the next target selection. Although time constraints seem to be important, they cannot, on their own, explain the differences in foraging strategies between the different selection modalities. Indeed, the results of Tagu \& Kristjánsson (2020) showed that observers were more likely to prioritize primed targets during gaze foraging, while the long inter-target times (about $685 \mathrm{~ms}^{1}$ in Tagu \& Kristjánsson, 2020) suggest that they should have prioritized proximity. At this point, it is therefore important to note that during gaze foraging participants are forced to be careful with their eye movements and fixations, to prevent accidental distractor selections, which is not the case during mouse or finger foraging. Hence, selecting targets with eye gaze may also affect visual exploration and oculomotor dynamics, and consequently the first step of the target selection process: the identification of the potential candidates for the next selection. We will revisit this issue since we will compare mouse and gaze foraging in this study.

Although past studies have identified conditions that would tilt the selection balance in favour of value, proximity or priming, all the studies mentioned above have also revealed large individual differences,

\footnotetext{
${ }^{1}$ All inter-target times here are extracted from previous research using a feature-based foraging task with 40 targets and 40 distractors, from 2 target types and two distractor types.
} 
suggesting that individuals have by-default internal biases towards value, proximity and priming, and are differentially sensitive to the effects of task difficulty, selection modality or time constraints. As an example, in Á. Kristjánsson et al. (2014) and later studies (Jóhannesson et al., 2017; Tagu \& Kristjánsson, 2020; Thornton et al., 2019, 2020) the differences in search strategy between featurebased and conjunction-based foraging were consistently found for most participants, but about one third of the participants - the so-called "super foragers" - systematically located the nearest target and frequently switched between target categories, even during difficult conjunction-based foraging. The mechanisms that differentiate these super foragers from other participants are however not fully understood. Jóhannesson et al. (2017) tested, for example, whether super foragers might have better executive functions, but their results did not reveal any differences in attentional or working memory capacity between normal and super foragers. Another possibility is that super foragers could show very strong internal biases towards proximity, that could override any influence of other factors favouring priming. Consistent with this assumption, observers can switch between conjunction targets when explicitly asked to do so (Wolfe et al., 2019), or when task demands require it (T. Kristjánsson et al., 2018), but only a few individuals (i.e., the "super foragers") spontaneously adopt this strategy. Similarly, in Wolfe et al. (2018), although the manipulation of target value strongly influenced the foraging patterns, there were clear individual differences. Some observers only selected the high-value targets, while others still selected some of the low-value targets before moving to a new display. Once again, these individual differences suggest that the former show a much stronger internal bias toward value than the latter. Nevertheless, it seems that these internal biases towards value, proximity and priming are intrinsically linked to the conditions and the task: by using three different visual search tasks (including single-target and multi-target visual search tasks), Clarke et al. (2020) have recently shown that individual differences in foraging strategy and performance were stable over repetitions of the same task, but that observers' strategies in one task were not predictive of their behaviour in other tasks. It is therefore possible that internal biases towards value, proximity and priming are task dependent. 
Here, our aim is to contribute to this growing field of research and to the identification of the factors

tilting the selection balance in favour of value, proximity or priming when determining which target to select next during visual foraging. Critically, we note that past research has largely focused on the second step of the target selection process, namely the selection of the best candidate for the next target selection, but that little is known about the mechanisms involved in the first step, the identification of a set of candidates (Wolfe et al., 2018). In the current study, we therefore investigated the mechanisms underlying target identification, notably by examining the potential involvement of eye movements during target selection. Current theories of visual orienting indicate that the identification process could either be achieved with overt shifts of attention and involve eye movements, or could be achieved with covert shifts of attention, not accompanied by eye movements (Posner \& Cohen, 1980; see also Hunt \& Kingstone, 2003). Although there is a large literature on eye movement behaviour during single-target visual search (for a review, see Eckstein, 2011), oculomotor dynamics during visual foraging are far less well known (Kosovicheva et al., 2020; Tagu \& Kristjánsson, 2020). Recently, Tagu \& Kristjánsson (2020) examined the influence of task difficulty and effector type on oculomotor dynamics during foraging, and showed that feature-based foraging is associated with higher fixation duration and smaller saccade amplitude than conjunction-based foraging, suggesting that easy search is associated with visual exploration in a focal mode while difficult search is associated with exploration in an ambient mode (Goldberg \& Kotval, 1999; Krejtz et al., 2016; Over et al., 2007; Unema et al., 2005; Velichkovsky et al., 2002). Importantly, these effects of task difficulty on oculomotor dynamics were accompanied by effects on foraging strategy (e.g., the order of target selections) and foraging dynamics (e.g., inter-target times), and were found both when observers

172 selected targets with a computer mouse and with eye gaze (Tagu \& Kristjánsson, 2020). In other words, 173 although there are some differences, foraging behaviour does not seem to vary much between mouse174 and gaze foraging in terms of foraging strategy, foraging dynamics or oculomotor dynamics. Critically, 175 as discussed above, during gaze foraging the target selection process is intrinsically linked to where 
participants look, and consequently, to eye movements. If participants do not covertly select a visual target prior to the eye movement, they are likely to select a distractor. Hence, similar foraging behaviour during mouse foraging (where participants are free to use overt orienting) and during gaze foraging (where covert orienting is much more probable) suggests that the identification of the set of candidates for the next target selection is achieved through covert orienting. Note however that the effect of task difficulty (feature-based versus conjunction-based foraging) has proven to be large and robust and may have overridden the potential effects of effector type on foraging behaviour (Tagu \& Kristjánsson, 2020). Moreover, although the study of Tagu \& Kristjánsson (2020) has cast light on how the selection balance between proximity and priming could influence oculomotor dynamics, the authors did not manipulate target value.

Here, for the first time, we used a feature-based foraging task to investigate the role of eye movements in target selection during visual foraging. More precisely, we examined how favouring overt orienting (in a mouse foraging task) or covert orienting (in a gaze foraging task) influences the competition between target value, proximity and priming, and therefore affects foraging strategy. In light of previous studies, we hypothesized that target value and proximity manipulations should strongly affect foraging behaviour (Wolfe et al., 2018), but that the results should not vary much between mouseand gaze foraging tasks (Tagu \& Kristjánsson, 2020), suggesting that eye movements play only a small role in target selection. Moreover, we expected to observe individual differences in foraging strategy, with considerable variation in the effects of target value and proximity across individuals, suggesting the presence of internal biases that guide target selection.

\section{Methods}

\subsection{Participants}

Twenty-four participants aged from 21 to 48 years (mean age $=28.6, S D=6.4$ ) were recruited from the Icelandic community. Fourteen were females, 21 were right-handed (self-reported) and 12 were right- 
eye-dominant (hole-in-card test, Durand \& Gould, 1910). Prior to their inclusion in the study, all participants received clear explanations about the procedure and gave their written informed consent. The study was completed in accordance with the requirements of the Icelandic bioethics committee and conformed with the ethical guidelines set out in the 1964 Declaration of Helsinki and its later amendments.

The sample size $(n=24)$ was determined prior to data collection and was based on recent studies with similar procedures where within-subject differences in foraging behaviour have been successfully measured (e.g., $n=16$ in Á. Kristjánsson et al., 2014; n = 21 in Kristjánsson and Kristjánsson, 2018; n = 24 in Tagu \& Kristjánsson, 2020; $n=12$ in Wolfe et al., 2018). Following data collection, we verified that this sample size would provide sufficient power to detect the main effects of block type, spatial organization and effector type, and the 2 (block type: value, no value) by 2 (spatial organization: random, patches) interaction, by conducting a power analysis using the "ss.power.wa.general()" function of the "Bias and Uncertainty Corrected Sample Size" (BUCSS) R package (Anderson et al., 2017). This function uses the F-value and sample size from a previous study to generate the necessary sample size needed to achieve the desired level of statistical power in the current study, for any type of effect using a within-subject ANOVA involving any number of factors. Here, we chose the previous study from our group (Tagu and Kristjánsson, 2020) that most closely resembles the current design. That study involved a 2 (block type: feature, conjunction) by 2 (effector type: mouse, gaze) repeatedmeasures analysis of variance conducted on a set of dependent variables, including the number of runs and inter-target times. Along with the sample size and alpha parameters from Tagu and Kristjánsson (2020), we used the F-values of the reported main effects of block type on the number of runs $(F[1,23]$ $\left.=93.1, p<.001, \eta_{p}{ }^{2}=.80\right)$ and of effector type on inter-target times $\left(F[1,23]=31.8, p<.001, \eta_{p}{ }^{2}=.58\right)$ as inputs for the BUCSS ss.power.wa.general() function. We used the by-default settings of assumed alpha (.05), level of assurance (.8) and desired power (.8). The analysis yielded a minimum sample size of 12. Because our study involves a more complex design ( $2 \times 2 \times 2$ within-subject design) than the $2 \times$ 
2 within-subject design of Tagu and Kristjánsson (2020), the doubled sample size $(n=24)$, should ensure that our study reaches the desired statistical power.

\subsection{Materials}

Stimuli were presented on a BenQ XL24211Z monitor (BenQ, Taipei, Taiwan) with a refresh rate of 144 $\mathrm{Hz}$ and a resolution of $1920 \times 1080$ pixels. The experiment took place in a dimly lit and soundproof room. The head of the participants was kept stable with a chin and forehead rest positioned $57 \mathrm{~cm}$ away from the monitor. Eye movements from the dominant eye were recorded using an EyeLink 1000 Plus (SR Research, Ontario, Canada) sampled at $2000 \mathrm{~Hz}$ and with an average spatial accuracy of $0.15^{\circ}$. The online saccade detection corresponded to an above-threshold velocity of $30 \% \mathrm{~s}$ and acceleration of $8000^{\circ} / \mathrm{s}^{2}$.

Each trial involved 108 coloured disks from six colour categories (18 stimuli per category) equalised in size $\left(0.5^{\circ}\right.$ diameter) and luminance $\left(20 \mathrm{~cd} / \mathrm{m}^{2}\right)$. Three of these categories (54 stimuli) were targets, and the other three (54 stimuli) were distractors. The colours were selected based on the six categories found for isoluminant colours in Witzel \& Gegenfurtner (2013) and refer to the basic colour terms "pink", "orange", "yellow", "green", "blue" and "purple". The stimuli were presented on a dark grey background with a luminance of $7 \mathrm{~cd} / \mathrm{m}^{2}$. As shown in the trial snapshot in Figure $1 \mathrm{~A}$, stimuli were distributed across a non-visible grid composed of twelve columns and nine rows and occupying $28^{\circ} \times$ $20^{\circ}$ of the visual field. The columns/rows of the grid were separated by an empty space of about $2^{\circ}$. However, the position of the stimuli within the grid was slightly jittered $\left( \pm 0.5^{\circ}\right)$ to create a less uniform appearance, modifying the size of the initial $2^{\circ}$ gap between stimuli. 


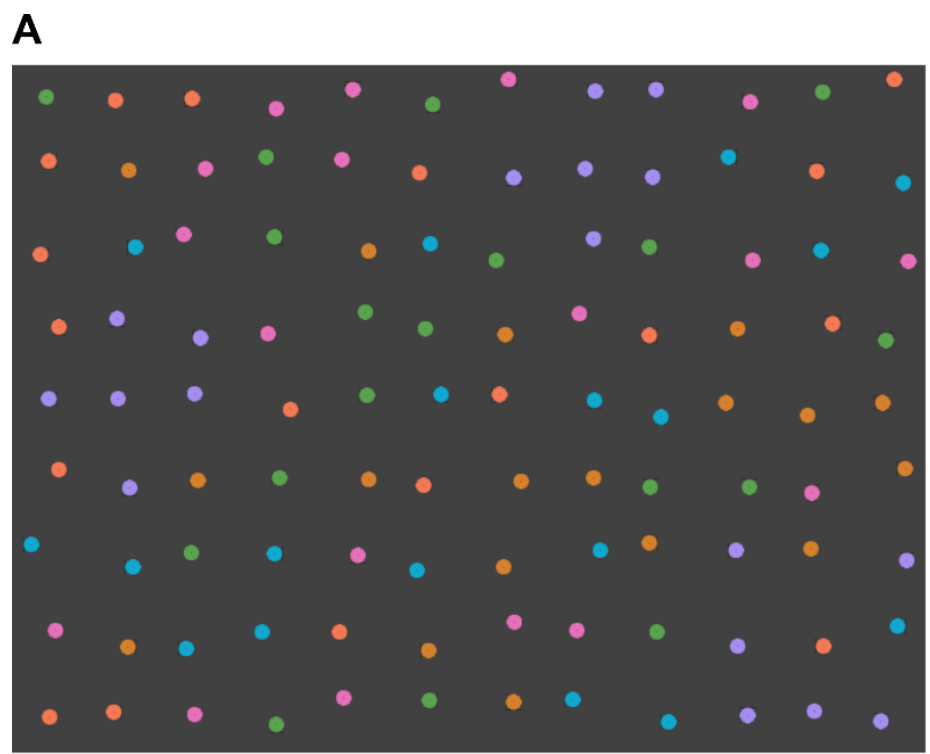

B

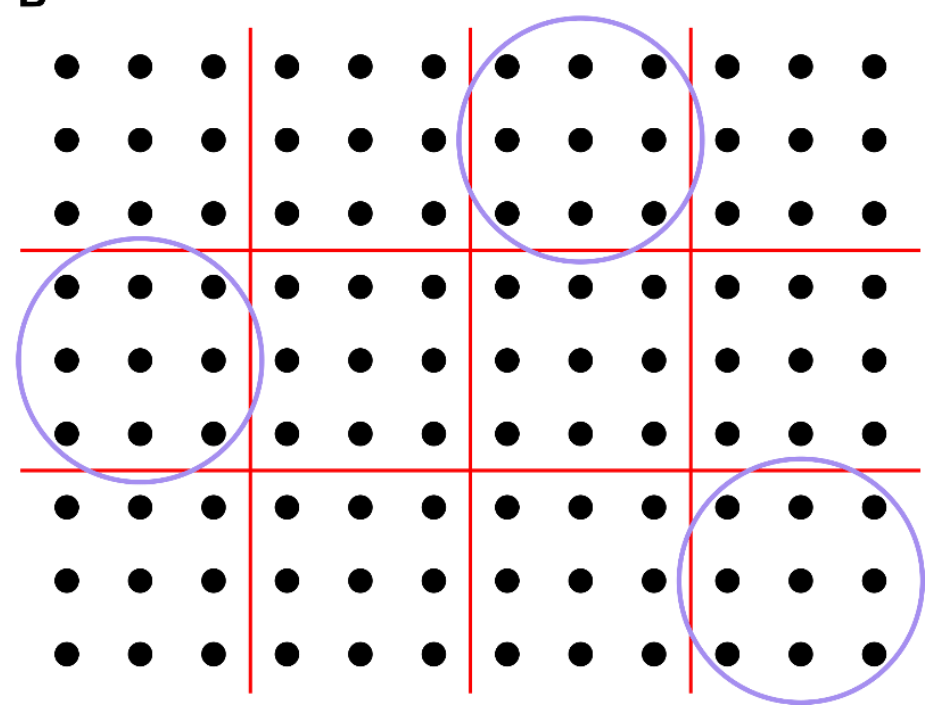

Figure 1. Example of a trial. A. Example of a trial from the patched-organization condition, with purple highvalue targets, orange and green low-value targets, and pink, blue and yellow distractors. B. Illustration of the grid used to assign targets to locations in the grid. The black points represent the target locations in the grid before the spatial jitter was applied, and the red lines show how the grid could be divided into 12 areas of 9 targets in the patched-organization condition. The purple circles indicate the areas selected for the patches of purple high-value targets for this particular trial.

\subsection{Procedure}

260 In four blocks of 20 trials each, participants had to select multiple instances of three target types (e.g., purple, orange and green) and ignore multiple instances of three distractor types (e.g., pink, yellow and blue). Target types were associated with a certain amount of points, and the trial ended once the 
participant had selected enough targets to reach a pre-specified number of points. The four blocks differed in the point value associated with the three target types (value block or no-value block) and by the effector used to select the targets (computer mouse or eye gaze). In the value blocks, participants had to earn 176 points to terminate the trials. Selecting targets from one of the target types (e.g., purple, the "high-value targets") made participants earn 6 points and selecting targets from the two other target types (e.g., orange and green, the "low-value targets") made them earn 2.5 points. The number of points for each target was chosen so that participants needed to select at least one item from each target type to complete the trials: if participants selected all 18 high-value targets, they would need to select at least 28 low-value targets to terminate the trials (which corresponds, e.g., to the 18 instances of one of the low-value categories, and half of the targets from the second low-value category). Participants were however not forced to select all the high-value targets, and they could select high- and low-value targets in any order, so that all combinations leading to 176 points would terminate the trials. Participants were told that they did not need to count the points because trials would automatically end once they had earned enough points. In the no-value blocks, all target types were associated with 1 point, and participants had to earn 54 points to terminate the trials. In other words, participants' task was to select all targets from all the three target categories. Participants had to complete each block type two times, one per effector type. During mouse foraging, participants were asked to select the targets by clicking on them with the left button of a computer mouse, while during gaze foraging they had to do so by fixating the targets with their eyes. Moreover, to manipulate target proximity, the spatial organization of the targets was manipulated within each block. Each block contained 10 trials where targets and distractors were randomly assigned to one of the 108 locations ("random organization"), intermixed with 10 trials where targets from one of the categories (which corresponded to the high-value targets in the value blocks) were distributed into three "patches". The idea here was to contrast target value and target proximity, since on "patched" trials prioritization of target value (i.e., selecting all the high-value targets first) would force observers to travel through the distant patches, while passing over quite a few of the low-value targets on the way. During the value 
blocks, the target category presented in patches was always the high-value category (e.g., purple). During the no-value blocks, the target category presented in patches was held constant for a given individual (e.g., blue). For the sake of clarity, in what follows and in the results section, we will refer to "high-value targets" for all targets presented in patches, irrespective of whether they were in the value block (in which they were indeed associated with high value) or in the no-value block (where they were not associated with a particular value). On trials organized with patches of high-value targets, the display was divided into twelve areas of nine targets (as illustrated in Figure 1B). The 18 high-value targets were randomly distributed in three of these areas, with two constraints: (1) there had to be at least two areas separating each patch of high-value targets, and (2) there had to be at least one highvalue target within each patch (i.e., it was not possible for the 18 high-value targets to be equally distributed in two of the three patches with the third one being composed of distractors and low-value targets). Once the high-value target locations were assigned, all other targets were randomly assigned to the 90 remaining locations in the grid. For example, Figure $1 \mathrm{~A}$ shows a trial in the patchedorganization condition, and Figure 1B shows three areas selected for the purple high-value targets of Figure $1 \mathrm{~A}$ (for a given trial). On trials with random spatial organization, all stimuli were randomly assigned to the 108 possible locations. In all conditions, the overall spatial layout and location of targets and distractors was generated independently on each trial.

Each block began with a nine-point calibration. The position of the dominant eye was checked before each trial, and if it was further than $0.75^{\circ}$ away (left, right, up or down) from the centre of the screen, the trial was cancelled and repeated later in the block, and a new calibration began before the next trial started. Moreover, each block was preceded by 2 training trials to familiarize the participant with the tasks. During the training, the trials were always from the random spatial organization condition. In all blocks, participants were instructed to finish the trials as fast as possible, without selecting any distractor. When a target was selected, it disappeared, while distractor selection led to an errormessage screen. As in previous research involving similar paradigms (e.g., Á. Kristjánsson et al., 2014; 
Tagu \& Kristjánsson, 2020; Thornton et al., 2019), we clearly explained to the participants before the experiment started that if they selected a distractor, the error message would be displayed and the entire foraging array with the 108 stimuli would be presented again later in the block. When a trial was successfully completed, a feedback screen appeared indicating the number of remaining trials in the block and the trial response time. Participants had to successfully complete 20 trials to terminate the blocks. The stimuli were surrounded by a $1.5^{\circ} \times 1.5^{\circ}$ rectangular interest area, and the stimulus selection was triggered when a mouse click (mouse foraging) or an eye fixation (gaze foraging) was detected in that area. The distance between items on screen and the strength of the spatial jitter applied to stimulus locations were chosen so that the interest areas never overlapped. During gaze foraging, target selection was triggered when an eye fixation longer than $150 \mathrm{~ms}$ was detected in the interest area, and distractor selection was triggered when an eye fixation longer than $350 \mathrm{~ms}$ was detected in the interest area. To make the gaze foraging task comparable to the mouse foraging task, it was important to provide observers with the possibility to both "miss" the targets they had not yet identified and briefly fixate some of the distractors before quickly shifting their gaze somewhere else once they had identified them as distractors (as they would do in a non-gaze-contingent task). The fixation times were chosen based on pre-tests run on two well-trained participants, where $150 \mathrm{~ms}$ turned out to be the optimal timing to prevent false detections of target selections during visual exploration without affecting fixation durations, and $350 \mathrm{~ms}$ the optimal timing to prevent omissions of distractor selections while enabling quick identification of the stimuli as distractors and continued exploration of the scene (see Tagu \& Kristjánsson, 2020, for similar manipulations).

The order of the tasks was counterbalanced so that half of the participants started with the no-value blocks and the other half with the value blocks. Moreover, half of them performed mouse foraging before gaze foraging while the other half did the reverse. The target identities were counterbalanced as well so that for half of the participants the targets were purple, orange and green among pink, yellow and blue distractors, while for the other participants the target and distractor identities were 
reversed. The identity of the high-value category was also counterbalanced, so that each colour was associated with the high-value targets for $1 / 6$ of the observers. To prevent any influence of the associations between target types and values from the value blocks to the no-value blocks, target and distractor identities were reversed for a given observer between the value- and no-value blocks but were held constant between the mouse and gaze foraging tasks.

\subsection{Data analysis}

In line with previous studies (e.g., Á. Kristjánsson et al., 2014; T. Kristjánsson et al., 2020; Tagu \& Kristjánsson, 2020), we assessed foraging strategy by measuring the number of "runs" on a given trial. A "run" refers to the sequential selection of targets of the same category. In our experiment, the number of runs could vary from 3 (one run per target category, such as when a participant never switches between target categories) to 54 (one run per target, such as when a participant always switches between target categories). Note that 54 could only be reached in the no-value blocks, as participants did not select all the targets in the value blocks. Hence, because of the differing number of target selections the number of runs may mechanically be higher in the no-value block than in the value block. To take this potential bias into account, we instead analysed the "proportional number of runs", that corresponds to the number of runs divided by the actual number of target selections on the trial. Furthermore, foraging dynamics were assessed by measuring inter-target times (the time in milliseconds that elapses between two successive target selections) and inter-target distances (distance in degrees of visual angle between two successive target selections). Note that the first and last target selections on a trial were removed from these analyses as they are known to show different dynamics than other selections (Á. Kristjánsson et al., 2019). To examine oculomotor dynamics during foraging, after having measured the total number of fixations on each trial, we filtered the eye movement data and only included fixations that were associated with target selection (i.e., eye fixations occurring just before the mouse click during mouse foraging or the ones triggering target selection during gaze foraging) and their subsequent saccades to the analysis (for similar analyses, see 
Tagu \& Kristjánsson, 2020). We then examined the average fixation duration and saccade amplitude for these "critical" eye movement data associated with target selection and search for the next target. In this article, we only present analyses run on these critical eye movement data, but all data from critical and non-critical eye movements are available on the Open Science Framework (OSF) page associated with this article at https://doi.org/10.31234/osf.io/48pzy.

All the dependent variables were analysed using repeated-measure analyses of variance (ANOVA). Within-subject factors included block type (no-value block, value block), effector (mouse, gaze) and spatial organization (random, patches). Moreover, we assessed the evidence in favour of the alternative hypothesis $H_{1}$ versus the null hypothesis $H_{0}$ using Bayes Factor analyses ${ }^{2}\left(B_{10}>1\right.$ is considered evidence in favour of $\mathrm{H}_{1}$, whereas $\mathrm{BF}_{10}<1$ is considered evidence in favour of $\mathrm{H}_{0}$ ) with the BayesFactor R package version 0.9.12-4.2 (Morey and Rouder 2018). The Bayes Factor analysis was performed using the default Jeffreys-Zellner-Siow priors (Rouder et al., 2012).

\section{Results}

\subsection{Foraging strategy: internal biases towards value, proximity and priming}

The first aim of this study was to confirm the existence of internal biases towards value, proximity and priming that would predict search strategy during visual foraging. Our main indicator of search strategy is the proportional number of runs, that can distinguish between conditions where individuals are biased towards priming (small number of runs) and conditions where individuals are biased towards proximity (high number of runs). As expected, manipulating target value strongly affected run patterns,

\footnotetext{
${ }^{2}$ Bayes Factors comprised between 1 and 3 yield anecdotal evidence for $\mathrm{H}_{1}$, values between 3 and 10 yield moderate evidence for $\mathrm{H}_{1}$, values between 10 and 30 bring strong evidence for $\mathrm{H}_{1}$, values between 30 and 100 yield very strong evidence for $\mathrm{H}_{1}$, and values above 100 yield extreme evidence for $\mathrm{H}_{1}$. In the same manner, Bayes Factors comprised between 0.33 and 1 yield anecdotal evidence for $\mathrm{H}_{0}$, values between 0.1 and 0.33 yield moderate evidence for $\mathrm{H}_{0}$, values between 0.033 and 0.1 yield strong evidence for $\mathrm{H}_{0}$, values between 0.01 and 0.033 yield very strong evidence for $\mathrm{H}_{0}$, and values below 0.01 yield extreme evidence for $\mathrm{H}_{0}$ (criteria proposed by Jeffreys, 1961, and modifed by Lee and Wagenmakers, 2014)
} 
the value block being associated with fewer (proportional) runs $(M=.35, S D=.09)$ than the no-value $\operatorname{block}\left(M=.41, S D=.10 ; F[1,23]=13.8, p<.001, \eta_{p}{ }^{2}=.37, B F_{10}>100\right.$; see Figure 2$)$. In other words, the run patterns suggest that individuals are biased towards proximity in the no-value block, while they are more biased towards priming in the value block. Importantly, run patterns also varied with spatial organization $\left(F[1,23]=188.7, p<.001, \eta_{p}^{2}=.89, B F_{10}>100\right)$, the proportional number of runs being higher on trials with random spatial organization $(M=.42, S D=.10)$ compared with trials organized with patches of targets $(M=.34, S D=.08)$. This suggests that the inclusion of patches of targets gives more weight to priming (as opposed to proximity) in the selection balance than when spatial organization is random. Although the interaction between spatial organization and block type is significant in the ANOVA $\left(F[1,23]=4.5, p<.05, \eta_{p}{ }^{2}=.16, B F_{10}=0.46\right)$, the observed data in Figure 2 suggests a rather weak interaction, and the Bayes Factor is in favour of the null hypothesis. Overall, this suggests that spatial organization and block type independently affect the selection balance.

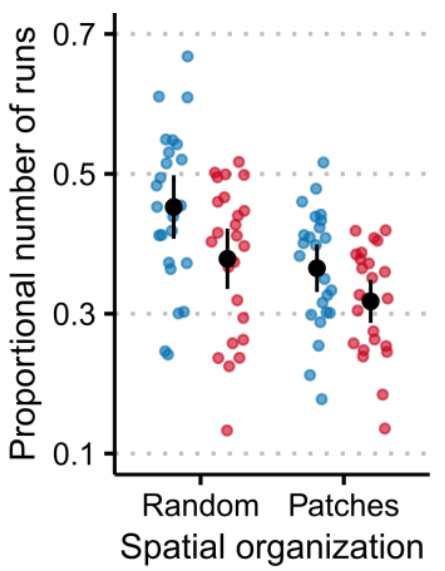

- No-value block • Value block

Figure 2. Interaction between the effects of block type and spatial organization on the proportional number of runs. Blue dots represent individual data points from the no-value block and red dots represent individual data points from the value block. Black dots and error bars represent the mean with 95\% confidence intervals.

Until now, we have reported results on proportional number of runs, that give a good contrast between prioritization of proximity (associated with many switches between target categories) and prioritization of priming (associated with few switches between target categories). However, these analyses do not provide a clear picture of how target value affects this competition between primed 
targets and proximal targets. Indeed, by definition, prioritization of value would mechanically involve prioritization of primed targets, as all high-value targets are from the same category. A more direct way to address whether target value affects foraging strategy consists in analysing the order in which high-value targets and low-value targets were selected. To this aim, we computed the "selection order", which ranges from 1 (first target being selected) to the total number of target selections on the trial (i.e., in no-value blocks, selection order always ranged from 1 to 54). Note that if an observer selects all the 18 high-value targets before the 36 low value targets on a given trial, the average selection order for the high-value targets of that trial would be $\frac{\sum_{n=1}^{18} n}{18}=9.5$. Similarly, selecting the high-value targets at random times during the trial would lead to an average selection order of $\frac{\sum_{n=1}^{54} n}{54}=$ 27.5. Remember that in the no-value blocks, "high-value targets" refer to the target category presented in patches in the non-random spatial organization condition, but these targets are not actually associated with a particular value. In Figure 3, we present the individual data on average selection order for high-value targets across trials. A quick look at the figure clearly shows that participants selected high-value targets earlier in the trial than low-value targets in the value block but did not do so in the no-value block. Interestingly, we also see considerable individual differences: while participants S01 to S03 clearly prioritized the high-value targets in the value block, participants S19 to S24 showed average selection orders over 20 , suggesting weak prioritization of high-value targets, and the other observers covered the range between these two extremes. Figure 3 also shows that in the no-value block, almost all participants selected high-value targets at random times during the trial, with average selection orders close to 27.5 . Moreover, in this analysis the prioritization of target proximity can also be assessed by comparing selection order of high-value targets during trials with random spatial organization and trials organized with patches of targets. Individual data from the novalue block show prioritization of proximity, with the target category presented in patches selected earlier during trials with a spatial organization in patches (blue triangles with dashed-line error bars in Figure 3) compared with trials with random spatial organization (blue disks with whole-line error bars 
in Figure 3). Note that this was not the case during the value blocks, where high-value targets were prioritized both when presented in patches and when randomly scattered among less valuable targets.

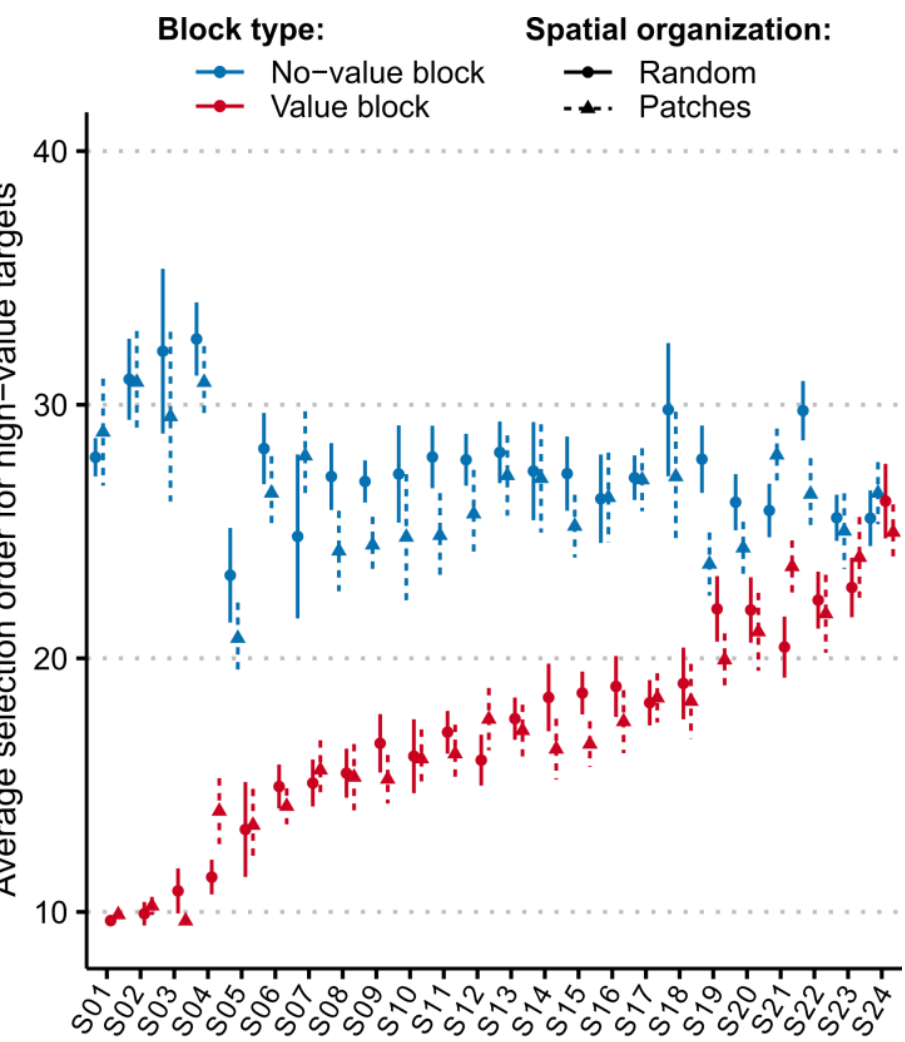

Participant ID

Figure 3. Individual data on selection order for high-value targets as a function of block type and spatial organization. Disks with whole-line error bars represent data from trials with random spatial organization, and triangles with dashed-line error bars represent data from trials organized with patches of targets. Blue lines represent data from the no-value block and red lines data from the value block. Participant IDs were ordered during the analysis, according to the average selection order. Error bars represent within-individual standard errors of the mean.

The ANOVA on selection order for high-value targets confirmed that selection order was significantly higher in the no-value block $(M=27.0, S D=2.1)$ than in the value block $(M=17.1, S D=4.2 ; F[1,23]=$ 81.7, $p<.001, \eta_{p}^{2}=.78, B F_{10}>100$ ). Furthermore, the analysis confirmed that spatial organization had only a small influence - if any - on selection order for high-value targets $\left(F[1,23]=7.7, p<.01, \eta_{p}{ }^{2}=\right.$ $\left..25, B F_{10}=0.21\right)$, with a Bayes Factor in favour of the null hypothesis. Similarly, the interaction between spatial organization and block type $\left(F[1,23]=7.5, p<.012, \eta_{p}{ }^{2}=.25, B F_{10}=0.34\right)$ suggests that the 
451

452

453

454

455

456

457

458

459

460

461

462

463

potential effect of spatial organization on selection order for high-value targets is only found in the novalue block (organization in patches: $M=26.4, S D=2.3$; random organization: $M=27.7, S D=2.2 ; p<$ .002 for Tukey HSD post-hoc test), not in the value block (organization in patches: $M=17.0, S D=4.2$; random organization: $M=17.2, S D=4.3 ; p=.89$ for Tukey HSD post-hoc test). Again, note however that the effect of spatial organization should be interpreted with caution, since even in the no-value block the effect is relatively weak and the Bayes factor is in favour of the null hypothesis. Overall, the results in Figure 3 suggest that foraging behaviour in the value block is driven by target value, while it is driven by target proximity in the no-value block. However, the analysis highlights strong individual differences in the influence of target value on selection order, which could be linked to different levels of individual internal biases towards value, proximity and priming.

\subsection{The role of overt and covert orienting in target selection during visual foraging}

The second aim of this study was to test the potential role of eye movements in target selection during visual foraging. Hence, this section is dedicated to comparing the mouse foraging task - in which observers were free to overtly orient to the next target during target selection - and the gaze foraging task - in which observers had to select targets by fixing their gaze on the current target and therefore needed to use covert orienting for identifying the next target during target selection.

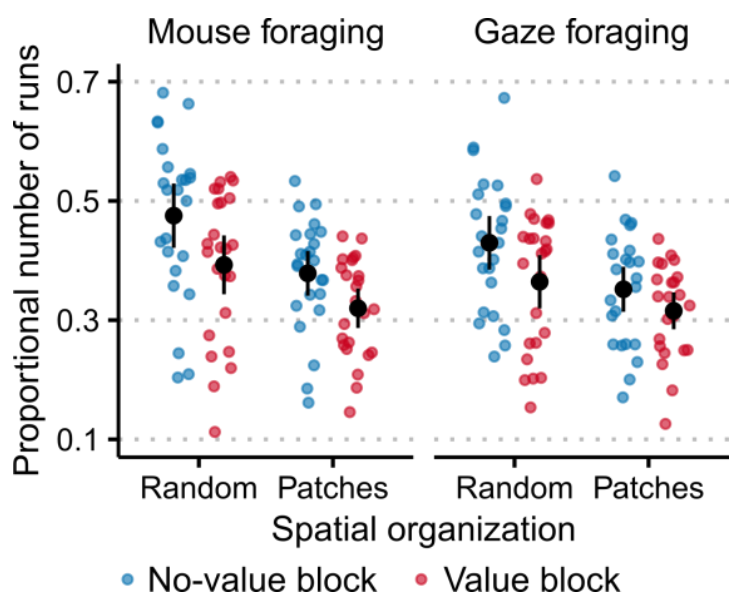



strategy. data points from the value block. The left panel represents data from the mouse foraging task, and the right panel represents data from the gaze foraging task. Black dots and error bars represent the mean with 95\% confidence intervals.

Interestingly, our main indicator of foraging strategy - the proportional number of runs - does not seem to vary much between mouse $(M=.39, S D=.09)$ and gaze $(M=.37, S D=.09)$ foraging $(F[1,23]=$ 4.1, $\left.p>.05, \eta_{p}^{2}=.15, B F_{10}=1.45\right)$, with only anecdotal evidence in favour of $\mathrm{H}_{1}$. Moreover, as Figure 4 shows, the ANOVA and Bayes Factor analyses suggest that the effector type used for target selection did not significantly modulate the effects of block type $\left(F[1,23]=1.4, p=.26, \eta_{p}{ }^{2}=.06, B F_{10}=.27\right)$ nor of spatial organization $\left(F[1,23]=5.8, p<.03, \eta_{p}^{2}=.20, B F_{10}=.34\right)$ on the proportional number of runs (note that the 3-way interaction is also non-significant: $F<1, B F_{10}=.26$ ). Overall, this analysis and Figure 4 importantly reveal that proportional run numbers do not vary between mouse- and gaze foraging, suggesting that blocking eye movements during target selection does not affect foraging

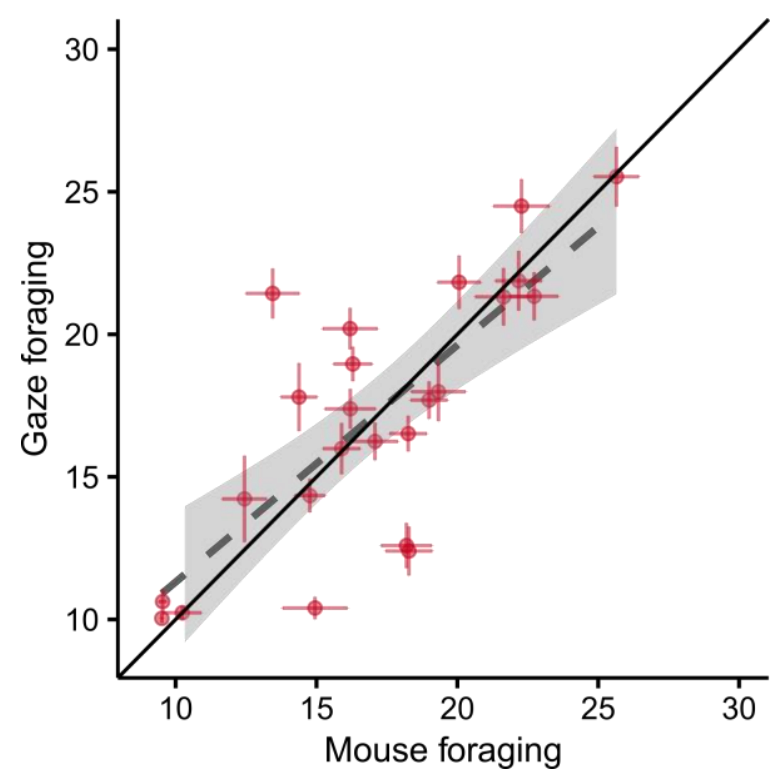
Figure 5. Correlation between selection order for high-value targets during mouse foraging (abscissa) and selection order for high-value targets during gaze foraging (ordinate). Red dots represent individual data points from the value block and error bars represent within-individual standard errors of the mean. The grey dashed line shows the best linear fit with 95\% confidence intervals, and the black plain line depicts what would 
494 Figure 5 shows little differences between mouse and gaze foraging in selection order for high-value 495 targets, as shown by the linear relationship between the selection order for high-value targets during 496 mouse foraging and the selection order for high-value targets during gaze foraging $(r=.77, p<.001$, $49795 \% \mathrm{Cl}=[.53, .90])$. In other words, the individual differences observed in the value block in Figure 3 498 are similar for mouse and gaze foraging, suggesting that individuals do not change their foraging 499 strategy with effector type. Again, this shows that preventing observers from overtly orienting to the next target during target selection does not change their foraging strategy.

501 

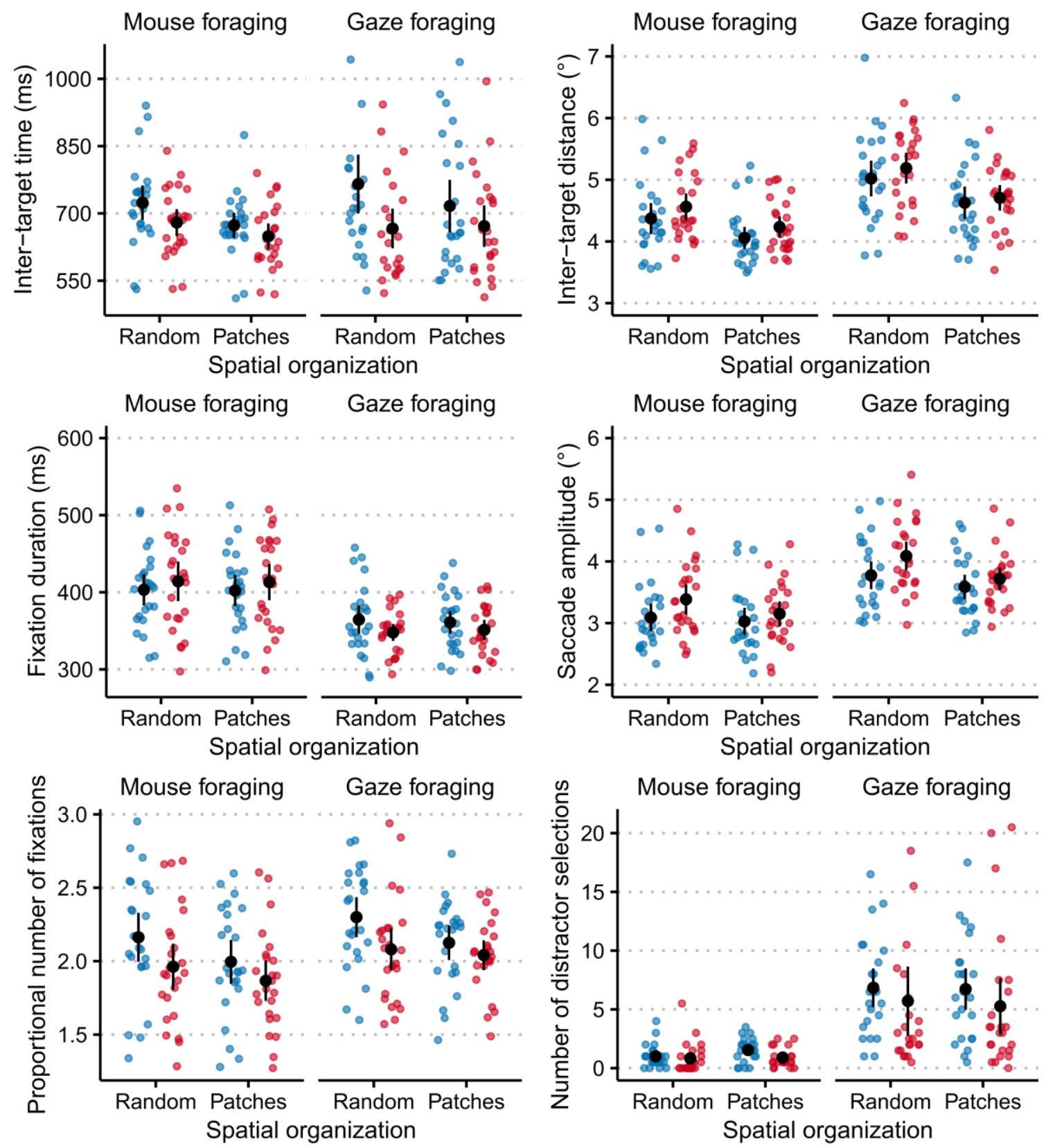

- No-value block • Value block

Figure 6. Results on inter-target times, inter-target distances, fixation durations, saccade amplitudes, proportional number of fixations and number of distractor selections, that overall show a cost associated with gaze foraging. Blue dots represent individual data points from the no-value block and red dots represent individual data points from the value block. In each plot, left panels represent data from the mouse foraging task, and right panels represent data from the gaze foraging task. Black dots and error bars represent the mean with 95\% confidence intervals.

510 It is important to note, however (as shown in Figure 6) that gaze foraging is also associated with more

511 variable inter-target times $\left(F[1,23]=34.7, p<.001, \eta_{p}{ }^{2}=.60, B F_{10}>100\right)$ and higher inter-target

512 distances $\left(F[1,23]=44.1, p<.001, \eta_{p}^{2}=.66, B F_{10}>100\right)$ than mouse foraging, suggesting that keeping 
the same foraging strategy with both effector types comes at a cost. Similarly, oculomotor dynamics revealed that gaze foraging was associated with more numerous eye fixations ${ }^{3}(F[1,23]=7.0, p<.02$, $\left.\eta_{p}^{2}=.23, B F_{10}>100\right)$ longer fixation duration $\left(F[1,23]=34.7, p<.001, \eta_{p}^{2}=.60, B F_{10}>100\right)$ and lower saccade amplitude $\left(F[1,23]=49.3, p<.001, \eta_{p}{ }^{2}=.68, B F_{10}>100\right)$ than mouse foraging (see Figure 6$)$. In the general discussion we suggest that these data indicate that gaze foraging is harder than mouse foraging (see also Tagu \& Kristjánsson, 2020, for similar discussion), and that although blocking overt orienting during target selection does not modulate foraging strategies, this comes at a cost. A final piece of evidence for the higher cost associated with gaze than mouse foraging is the higher number of distractor selections made by observers during gaze $(M=6.1, S D=4.4)$ than mouse foraging $(M=$ 1.1, $S D=0.7 ; F[1,23]=35.6, p<.001, \eta_{p}^{2}=.61, B F_{10}>100$; see Figure 6).

\section{Discussion}

Target selection during visual foraging is accomplished with two successive processes: first the identification of a set of candidate targets for the next selection, and second the selection of the best option among these candidates. Past research has mainly focused on the second step and has been aimed at identifying the factors influencing the identity of the target being selected, which notably include internal biases towards target value, proximity and priming (see e.g., Wolfe et al., 2018). However, little attention has been paid to the first step of the target selection process, namely the factors influencing the identification of a set of candidates. Here, our aims were (1) to confirm that individual differences found on run patterns during visual foraging could be explained by internal biases towards target value, proximity and priming, using a single feature-foraging task, and (2) to examine the respective contributions of overt and covert orienting to the first step of target selection, the identification of a set of candidates for the next selection.

\footnotetext{
${ }^{3}$ Note that, as for the number of runs, analyses were actually run on the "proportional" number of eye fixations, that corresponds to the number of fixations divided by the number of target selections in the trial.
} 


\subsection{Balancing value, proximity and priming}

Critically, our results show that both target value and proximity strongly shape foraging behaviour, as shown by the main effects of the target value and spatial organization manipulations on almost all dependent variables. Moreover, the results found on selection order suggest some hierarchization between value and proximity: when all items have the same value, participants mainly prioritize proximal targets over primed targets, but when targets have differing values, participants tend to prioritize more valuable targets. This result is consistent with previous research showing strong prioritization of high-value targets, even when they are rare (e.g., Wolfe et al., 2018). One could however argue that the effect of block type in our study could be driven more by the difference in task instructions between the blocks (earn a prespecified number of points in the value block or select all the targets on screen in the no-value block) than by the difference in target value manipulations. The results of Á. Kristjánsson et al. (2020) however suggest that this is not very likely since they found no difference in foraging strategy between an exhaustive foraging task and a non-exhaustive foraging task. Moreover, Wolfe et al. (2019) found slightly different foraging strategies in the two tasks showing that observers asked to select all available targets tend to prioritize primed targets (i.e., they foraged in few long runs) compared with observers who could move to a new display before having selected all available targets (who foraged in many runs of short length). So, although we acknowledge that

554 these differences in task instructions may have slightly affected the results, we argue that the 555 instruction we gave in the no-value block (select all available targets to terminate the trial) was more 556 likely to trigger run behaviour similar to what we observed in the value block (i.e., few long runs). Observing an effect of block type on run behaviour in these conditions therefore highlights how large and robust the influence of target value on foraging strategy is. 
Interestingly, using single-target visual search, Nowakowska et al. (2021) recently investigated whether individual differences in visual search performance could be explained by differences in motivation. In one of their experiments, the manipulation of motivation was achieved with reward manipulations. Participants had to complete two blocks of trials, and after having completed the first block, participants from the reward group were told that they would receive more money if they improved their performance in the second block compared to the first block, while participants from the flatpayment group were only asked to be as fast and as accurate as possible. The results however revealed that although both groups improved their performance from the first to the second block, this improvement was not larger for the reward group than for the flat-payment group. The authors therefore conclude that practice, rather than motivation and reward, makes participants more efficient searchers. This absence of a reward effect may appear contradictory with the strong effect of block type in the present study. However, before comparing the two experiments, it is important to delineate the methodological differences that may explain the different results. Firstly, while Nowakowska et al. (2021) manipulated explicit reward (the actual money earned by the participants after having completed the experiment), we here manipulated target value: in the value block, some targets were worth more points than others, but participants did not earn any more money in the value block compared with the no-value block. Actually, in the current study participants were equally motivated in both blocks of trials. Note however that the absence of reward does not necessarily means less motivation. On the contrary, here, after each trial, participants saw a feedback screen showing the trial response time and were therefore strongly motivated to "beat their score" on the subsequent trials (a bit like in a video game). Secondly, while the reward manipulation in Nowakowska et al. (2021) involves a between-subject design, the target value manipulation we used involves a within-subject design. This is important and may partly explain the differences between the two studies. Thirdly, and maybe most importantly, in Nowakowska et al. (2021) observers had to find a single target per trial, while the current study involves many target selections per trial. Recent studies have shown that the results from single-target visual search do not necessarily apply to visual foraging 
tasks, and vice versa (see e.g., T. Kristjánsson et al., 2020; Ólafsdóttir et al., 2020). To our knowledge, reward manipulations and differences in motivation have actually never been tested in visual foraging tasks, and future studies are needed to test whether the results from Nowakowska et al. (2021) could apply to foraging behaviours. Similarly, the potential generalizability of the foraging effects described here to single-target visual search should be addressed in future studies.

A last line of thought that we would like to highlight regarding the interactions between value, proximity and priming comes from previous research on single-target search suggesting that priming of features boosts both the speed of the search, as evidenced by lower reaction times with repeated features (Goolsby \& Suzuki, 2001; Maljkovic \& Nakayama, 1994; Treisman, 1992), and perceptual sensitivity, as evidenced by higher accuracy, or $d^{\prime}$, with repeated features (Ásgeirsson et al., 2015; Geyer et al., 2010; Sigurdardottir et al., 2008). This research therefore reveals that single-target search is more efficient when based on priming of previous features than when based on spatial location (for recent reviews, see Á. Kristjánsson \& Ásgeirsson, 2019; Wolfe \& Horowitz, 2017). As discussed in the introduction, foraging studies have however revealed that the prioritization of priming over spatial location - or proximity - depends on other factors, such as task difficulty (Á. Kristjánsson et al., 2014), and that during easy feature-based foraging, favouring proximity can be equally efficient, or even more efficient, than favouring priming. Here, we show that target value is also an important factor to consider, and that value tilts the selection balance towards priming, even during an easy feature-based foraging task. Hence, our results (notably on inter-target times) replicate the increase in search speed with priming, previously found in single-target visual search, using multitarget foraging. Future studies may reveal whether value and priming also boost perceptual sensitivity during visual foraging, for example by using a paradigm allowing assessment of perceptual performance. One possibility could be for example the addition of an adjustment task where observers judge the exact colours of the target they just selected on a colour wheel. If priming boosts perceptual sensitivity during foraging, 
performance on the adjustment task should be better when the adjustment is made within a run than when it is made just after a switch.

\subsection{Individual differences and internal biases}

As discussed in the introduction, individual differences are often observed in visual foraging tasks (Clarke et al., 2020; Jóhannesson et al., 2017; Á. Kristjánsson et al., 2014; Tagu \& Kristjánsson, 2020; Wolfe et al., 2018). Individuals show, by default, internal biases towards value, proximity and priming (Wolfe et al., 2018). There is however evidence that these internal biases can be weighted by factors such as task difficulty (e.g., Á. Kristjánsson et al., 2014), time constraints (e.g., Thornton et al., 2020) or selection modality (e.g., Thornton et al., 2019). Importantly, the current results (1) confirm the existence of different internal biases towards value, proximity and priming, as observed in Figure 3, and (2) show that these internal biases can be weighted by the target value manipulation, but that the effector manipulation does not affect foraging strategy much. Consistent with Clarke et al. (2020), this suggests that these by-default internal biases - or individual differences - are stable over repetitions of the same task (i.e., repetition of a given block type with both effectors), but that an observer's foraging behaviour in one task (e.g., no-value block) is not predictive of their behaviour in another task (e.g., value block). Again, we do, however, see some sort of hierarchization between these internal biases. Although the magnitude of the effect of block type on selection order differs between individuals, our results clearly show that when targets are associated with differing value, all observers tilt their selection balance towards value (rather than proximity). Moreover, the effect of target proximity, found on selection order in the no-value block, disappears in the value block, as if target value had "won" the competition. These results therefore reinforce the assumption that whatever the strength of the observer's default internal biases, target value seems to weigh higher in the selection balance than proximity and priming (see also Wolfe et al., 2018; Wolfe \& Horowitz, 2017). Importantly, now that the current study has cast light on how internal biases towards value, proximity and priming could affect target selection and lead to individual differences in foraging behaviour, future studies 
637 from visual and computer science could use these data to build new computational models of foraging

638 behaviour for precisely predicting the foraging behaviour of individuals according to the task 639 characteristics. Such future studies could make an important contribution to the young field of human 640 visual foraging.

\subsection{Overt and covert orienting}

643

Another crucial result is that our effector type manipulation did not have much influence on run patterns and selection order, suggesting that observers kept foraging using the same strategy during mouse and gaze foraging. With this manipulation, our aim was to investigate the role of eye movements in the first step of the target selection process, namely the identification of a set of candidates for the next selection. During mouse foraging, observers were free to overtly orient within the display, whereas this behaviour was restricted during gaze foraging where observers were more likely to use covert orienting to avoid involuntary distractor selections. The results however indicate that favouring overt or covert orienting for the identification of a set of candidates does not have much influence on the selection of one of these candidates. On the contrary, the results suggest that observers modified their foraging and oculomotor dynamics (e.g., reaction times, fixation durations) in order to continue foraging with the same strategy with both effectors. Namely, gaze foraging was associated with a higher number of fixations, larger saccade amplitude and shorter fixation durations than mouse foraging. These results are consistent with visual exploration in a focal mode during mouse foraging and an ambient mode during gaze foraging (Goldberg \& Kotval, 1999; Krejtz et al., 2016; Over et al., 2007; Unema et al., 2005; Velichkovsky et al., 2002). This is surprising as previous research (Tagu \& Kristjánsson, 2020) did not reveal any differences in oculomotor dynamics between mouse and gaze foraging. Note however that the study of Tagu \& Kristjánsson (2020) involved a comparison between feature-based and conjunction-based foraging, and that the effects linked to this factor were so strong that they might have masked the potential effects of other manipulations. Here, by using feature- 
based foraging only, our paradigm may have been far more sensitive to the effector-type manipulation, revealing differences in oculomotor dynamics between mouse and gaze foraging.

Tagu \& Kristjánsson (2020) showed that easy feature-based foraging was associated with the focal mode (long fixation durations and small saccade amplitudes), and difficult conjunction-based foraging was associated with the ambient mode (short fixations and large amplitudes) of visual exploration. Hence, the association of gaze foraging with the ambient mode found here may suggest that gaze foraging was a more difficult task than mouse foraging. Consistent with this assumption, the results of Tagu \& Kristjánsson (2020) revealed that participants made more distractor selections and were more likely to select items in runs (i.e., to prioritize priming over proximity) during gaze-conjunction foraging than during mouse-conjunction foraging. During both mouse and gaze foraging, participants were more likely to prioritize priming over proximity during conjunction-based foraging, compared with feature-based foraging. Here, in an easier feature-based foraging paradigm, participants were overall more likely to prioritize proximity over priming (especially in the no-value block), as shown by the very high number of runs. However, it seems that continuing to prioritize proximity over priming during more difficult gaze foraging came at a cost, as shown notably by the higher number of distractor selections, the higher number of fixations, and exploration in an ambient mode (which by definition would not favour a strategy consisting of locating the nearest target, see Tagu \& Kristjánsson, 2020). All in all, the current results and past evidence therefore suggest that the most efficient and least costly strategy during gaze foraging would be to forage in long runs of selecting items from the same category and to prioritize priming over proximity (see also Thornton et al., 2019).

Importantly, our results suggest that eye movements are not required for completing the first step of the target selection process, namely the identification of a set of candidates for the next selection. In other words, being able to overtly orient (mouse foraging) or not (gaze foraging) during target selection does not seem to modulate behaviour at the second step, the selection of the best candidate target. 
688 It is however important to note that in our tasks, there is no guarantee that observers used overt 689 orienting during mouse foraging. Even if the paradigm allowed them to overtly orient, they could, of 690 course, covertly attend to peripheral targets to identify a set of candidates for the next selection. 691 Consistent with this assumption, our results revealed that observers made fewer fixations and

692

693

694

695

696

697

698

699 saccades during mouse than during gaze foraging. Moreover, the fact that being able to overtly orient or not does not change foraging strategy suggests that covert attention is involved at the first step of the target selection process, both during mouse and gaze foraging. Although the effector manipulation did not influence foraging strategy, it is worth noting that it did affect oculomotor dynamics. Moreover, gaze foraging, where it is more difficult to use overt orienting, comes at a cost, with higher inter-target times and distances and more distractor selections. Hence, our results suggest that overt orienting is not involved in the identification of candidates for the next selection, but that it is involved in the selection of the best option among these candidates. Future research may examine more specifically the potential roles of overt and covert orienting in the target selection process, focusing in particular on the importance of covert attention for target selection. Preview methods (Castelhano \& Henderson, 2007), which consist in flashing the search display for a fraction of seconds only, may for example be another interesting tool to consider for inducing covert orienting during visual foraging.

In addition to the potential involvement of overt and covert orienting, another fundamental difference between mouse and gaze foraging is that during mouse foraging, observers can anticipate the next selection with eye movements. While selecting the current target, observers may already have identified a set of candidates for future selections, for example with covert orienting, and may have already initiated the planning of an overt movement towards the target they want to select next (Kosovicheva et al., 2020; Thornton \& Horowitz, 2004). In four experiments, Kosovicheva et al. (2020) indeed recently demonstrated that during visual foraging the selection of the next target can be planned ahead, in parallel with the current selection. But if such anticipation is possible during mouse foraging, the gaze foraging paradigm prevented observers from anticipating the next selection with 
their eyes. In the current study, the distance between eye position and current target position at the time of target selection is on average $2.07^{\circ}\left(S D=0.71^{\circ}\right)$ during mouse foraging, while it is only $0.73^{\circ}$ $\left(S D=0.11^{\circ}\right)$ during gaze foraging. In other words, participants' eyes may already be "on their way" towards the next target during mouse foraging, while this was not the case during gaze foraging. Hence, although the effector-type manipulation revealed that the identification of candidates for the next selection can be performed through covert orienting, it also confirms that the selection of the next target can be planned in advance, in parallel with the current selection. All in all, our results therefore suggest that overt orienting is not needed for identification of a set of candidate targets for the next selection, but that it may be necessary for efficient selection of the best option among these candidates. This last assumption would moreover explain why the effector-type manipulation modified foraging and oculomotor dynamics without influencing foraging strategy. This assumption however needs to be addressed in future foraging studies specifically designed to test the involvement of overt and covert orienting at the different stages of the target selection process.

\subsection{Summary and conclusions}

For the first time, we have assessed how target value, proximity and priming affect foraging strategy, foraging dynamics and oculomotor dynamics in a multi-target visual search task. Moreover, by contrasting mouse and gaze foraging, we have investigated how differently favouring overt and covert orienting could affect foraging behaviour. Our results critically reveal some hierarchization between the three forces that tilt the selection balance as the presence of targets with differing values overrides any potential influence of target proximity on foraging behaviour. Moreover, we have shown that target value modifies all aspects of foraging behaviour by influencing foraging strategy, foraging dynamics and oculomotor dynamics. Finally, by contrasting mouse and gaze foraging we have shown that overt orienting is not necessary for identifying candidates for the next selection, but that it may be involved in the selection of the best option among these candidates. 
Funding: This work was supported by the Icelandic Research Fund (grant number 206744-051) and a grant from the University of Iceland Research Fund.

\section{Declarations of interest: None.}

Data availability statement: All data, mean tables and ANOVA tables have been made publicly available via the Open Science Framework and can be accessed at https://doi.org/10.17605/OSF.IO/E7CND.

Acknowledgements: The authors thank Alasdair Clarke, Johan Wagemans, Jeremy Wolfe and an anonymous reviewer for very helpful comments and suggestions, and thank Kolfinna Thórisdóttir for her help with data collection.

Anderson, S. \& Kelley, K., Maxwell, S. E. (2017). Sample size planning for more accurate statistical power: A method correcting sample effect sizes for uncertainty and publication bias. Psychological Science, 28(11), 1547-1562. https://doi.org/10.1177/0956797617723724

Ásgeirsson, Á. G., Kristjánsson, Á., \& Bundesen, C. (2015). Repetition priming in selective attention: A TVA analysis. Acta Psychologica, 160, 35-42. https://doi.org/10.1016/j.actpsy.2015.06.008

Cain, M. S., Vul, E., Clark, K., \& Mitroff, S. R. (2012). A bayesian optimal foraging model of human visual search. Psychological Science, 23(9), 1047-1054. https://doi.org/10.1177/0956797612440460

Castelhano, M. S., \& Henderson, J. M. (2007). Initial scene representations facilitate eye movement guidance in visual search. Journal of Experimental Psychology: Human Perception and Performance, 33(4), 753-763. https://doi.org/10.1037/0096-1523.33.4.753

Clarke, A. D., Irons, J. L., James, W., Leber, A. B., \& Hunt, A. R. (2020). Stable individual differences in 
strategies within, but not between, visual search tasks. Quarterly Journal of Experimental Psychology, 1747021820929190. https://doi.org/10.1177/1747021820929190

Durand, A. C., \& Gould, G. M. (1910). A method of determining ocular dominance. Journal of the $\begin{array}{lll}\text { American } & \text { Medical } & \text { Association, }\end{array}$ https://doi.org/10.1001/jama.1910.04330050007004

Eckstein, M. P. (2011). Visual search: A retrospective. Journal of Vision, 11(5), 1-36. https://doi.org/10.1167/11.5.14

Fougnie, D., Cormiea, S. M., Zhang, J., Alvarez, G. A., \& Wolfe, J. M. (2015). Winter is coming: How humans forage in a temporally structured environment. Journal of Vision, 15(11), 1-1. https://doi.org/10.1167/15.11.1

Geyer, T., Zehetleitner, M., \& Müller, H. J. (2010). Contextual cueing of pop-out visual search: When context guides the deployment of attention. Journal of Vision, 10(5), 20-20. https://doi.org/10.1167/10.5.20

Goldberg, J. H., \& Kotval, X. P. (1999). Computer interface evaluation using eye movements: Methods and constructs. International Journal of Industrial Ergonomics, 24(6), 631-645. https://doi.org/10.1016/S0169-8141(98)00068-7

Goolsby, B. A., \& Suzuki, S. (2001). Understanding priming of color-singleton search: Roles of attention at encoding and "retrieval". Perception \& Psychophysics, 63(6), 929-944. https://doi.org/10.3758/BF03194513

Hills, T. T., Kalff, C., \& Wiener, J. M. (2013). Adaptive Lévy Processes and Area-Restricted Search in Human Foraging. PLOS ONE, 8(4), e60488. https://doi.org/10.1371/journal.pone.0060488

Hulleman, J., \& Olivers, C. N. L. (2017). The impending demise of the item in visual search. Behavioral and Brain Sciences, 40. https://doi.org/10.1017/S0140525X15002794

Hunt, A. R., \& Kingstone, A. (2003). Covert and overt voluntary attention: Linked or independent? Cognitive Brain Research, 18(1), 102-105. https://doi.org/10.1016/j.cogbrainres.2003.08.006 Jeffreys, H. (1961). Theory of probability, 3rd Edition. Oxford, United Kingdom: Oxford University Press. 
791

Jóhannesson, Ó. I., Kristjánsson, Á., \& Thornton, I. M. (2017). Are Foraging Patterns in Humans Related to Working Memory and Inhibitory Control? Japanese Psychological Research, 59(2), 152-166. https://doi.org/10.1111/jpr.12152

Kosovicheva, A., Alaoui-Soce, A., \& Wolfe, J. M. (2020). Looking ahead: When do you find the next item in foraging visual search? Journal of Vision, 20(2), 3-3. https://doi.org/10.1167/jov.20.2.3

Krejtz, K., Duchowski, A., Krejtz, I., Szarkowska, A., \& Kopacz, A. (2016). Discerning Ambient/Focal Attention with Coefficient K. ACM Trans. Appl. Percept., 13(3), 11. https://doi.org/10.1145/2896452

Kristjánsson, Á. (2015). Reconsidering Visual Search. I-Perception, 6(6), 2041669515614670. https://doi.org/10.1177/2041669515614670

Kristjánsson, Á., \& Ásgeirsson, Á. G. (2019). Attentional priming: Recent insights and current controversies. Current Opinion in Psychology, 29, 71-75. https://doi.org/10.1016/j.copsyc.2018.11.013

Kristjánsson, Á., Björnsson, A. S., \& Kristjánsson, T. (2020). Foraging with Anne Treisman: Features versus conjunctions, patch leaving and memory for foraged locations. Attention, Perception, \& Psychophysics, 82(2), 818-831. https://doi.org/10.3758/s13414-019-01941-y

Kristjánsson, Á., Jóhannesson, Ó. I., \& Thornton, I. M. (2014). Common Attentional Constraints in Visual Foraging. PloS One, 9(6), e100752. https://doi.org/10.1371/journal.pone.0100752

Kristjánsson Á., Ólafsdóttir I.M., Kristjánsson T. (2019). Visual foraging tasks provide new insights into the orienting of visual attention: Methodological considerations. In: Pollmann S. (Ed.), Spatial Learning and Attention Guidance. Neuromethods, vol 151 (pp. 3-21). Humana Press: New York, NY. https://doi.org/10.1007/7657_2019_21

Kristjánsson, T., \& Kristjánsson, Á. (2018). Foraging through multiple target categories reveals the flexibility of visual working memory. Acta Psychologica, 183, 108-115. https://doi.org/10.1016/j.actpsy.2017.12.005

Kristjánsson, T., Thornton, I. M., Chetverikov, A., \& Kristjánsson, Á. (2020). Dynamics of visual attention 


$$
\text { revealed }
$$

in

foraging

tasks.

Cognition,

194,

104032. https://doi.org/10.1016/j.cognition.2019.104032

Kristjánsson, T., Thornton, I. M., \& Kristjánsson, Á. (2018). Time limits during visual foraging reveal flexible working memory templates. J Exp Psychol Hum Percept Perform, 44(6), 827-835.

821

Lee, M. D., \& Wagenmakers, E. J. (2014). Bayesian cognitive modeling: a practical course. Cambridge, United Kingdom: Cambridge University Press.

823

824

825

Maljkovic, V., \& Nakayama, K. (1994). Priming of pop-out: I. Role of features. Memory \& Cognition, 22(6), 657-672.

Morey, R. D., \& Rouder, J. N. (2018). Package "BayesFactor". R package version 0.9.12-4.2. Available at https://cran.r-project.org/web/packages/BayesFactor/index.html

Nowakowska, A., Clarke, A. D., von Seth, J., \& Hunt, A. R. (2021). Search strategies improve with practice, but not with time pressure or financial incentives. Journal of Experimental Psychology: Human Perception and Performance.

Ólafsdóttir, I. M., Gestsdóttir, S., \& Kristjánsson, Á. (2020). Age differences in foraging and executive functions: A cross-sectional study. Journal of Experimental Child Psychology, 198:104910. https://doi.org/10.1016/i.jecp.2020.104910

Over, E. A. B., Hooge, I. T. C., Vlaskamp, B. N. S., \& Erkelens, C. J. (2007). Coarse-to-fine eye movement strategy in visual search. Vision Research, 47(17), 2272-2280. https://doi.org/10.1016/j.visres.2007.05.002

Palmer, J. (1994). Set-size effects in visual search: The effect of attention is independent of the stimulus for simple tasks. Vision Research, 34(13), 1703-1721. https://doi.org/10.1016/00426989(94)90128-7

Posner, M. I., \& Cohen, Y. (1980). 14 Attention and the Control of Movements. In G. E. S. and J. Requin (Ed.), Advances in Psychology (Vol. 1, pp. 243-258). North-Holland. http://www.sciencedirect.com/science/article/pii/S0166411508619494

Rouder, J. N., Morey, R. D., Speckman, P. L., \& Province, J. M. (2012). Default Bayes factors for ANOVA 
843

designs. Journal of Mathematical Psychology, 56(5), 356-374. https://doi.org/10.1016/j.jmp.2012.08.001

Sigurdardottir, H. M., Kristjánsson, Á., \& Driver, J. (2008). Repetition streaks increase perceptual sensitivity in visual search of brief displays. Visual Cognition, 16(5), 643-658. https://doi.org/10.1080/13506280701218364

Tagu, J., \& Kristjánsson, Á. (2020). Dynamics of attentional and oculomotor orienting in visual foraging tasks. Quarterly Journal of Experimental Psychology, 1747021820919351. https://doi.org/10.1177/1747021820919351

Thornton, I. M., de'Sperati, C., \& Kristjánsson, Á. (2019). The influence of selection modality, display dynamics and error feedback on patterns of human foraging. Visual Cognition, 27(5-8), 626-648. https://doi.org/10.1080/13506285.2019.1658001

Thornton, I. M., \& Horowitz, T. S. (2004). The multi-item localization (MILO) task: Measuring the spatiotemporal context of vision for action. Perception \& Psychophysics, 66(1), 38-50. https://doi.org/10.3758/BF03194859

Thornton, I. M., Nguyen, T. T., \& Kristjánsson, Á. (2020). Foraging tempo: Human run patterns in multiple-target search are constrained by the rate of successive responses. Quarterly Journal of Experimental Psychology, 1747021820961640. https://doi.org/10.1177/1747021820961640

Treisman, A. M. (1992). Perceiving and re-perceiving objects. American Psychologist, 47(7), 862-875. https://doi.org/10.1037/0003-066X.47.7.862

Treisman, A. M., \& Gelade, G. (1980). A feature-integration theory of attention. Cognitive Psychology, 12(1), 97-136. https://doi.org/10.1016/0010-0285(80)90005-5

Unema, P. J. A., Pannasch, S., Joos, M., \& Velichkovsky, B. M. (2005). Time course of information processing during scene perception: The relationship between saccade amplitude and fixation duration. Visual Cognition, 12(3), 473-494. https://doi.org/10.1080/13506280444000409

Velichkovsky, B. M., Rothert, A., Kopf, M., Dornhöfer, S. M., \& Joos, M. (2002). Towards an expressdiagnostics for level of processing and hazard perception. Transportation Research Part F: Traffic 
Psychology and Behaviour, 5(2), 145-156. https://doi.org/10.1016/S1369-8478(02)00013-X

Witzel, C., \& Gegenfurtner, K. R. (2013). Categorical sensitivity to color differences. Journal of Vision, 13(7), 1-33. https://doi.org/10.1167/13.7.1

Wolfe, J. M. (2010). Visual search. Current Biology, 20(8), R346-R349. https://doi.org/10.1016/j.cub.2010.02.016

Wolfe, J. M. (2013). When is it time to move to the next raspberry bush? Foraging rules in human visual search. Journal of Vision, 13(3), 1-17. https://doi.org/10.1167/13.3.10

Wolfe, J. M., Aizenman, A. M., Boettcher, S. E. P., \& Cain, M. S. (2016). Hybrid foraging search: Searching for multiple instances of multiple types of target. Vision Research, 119, 50-59. https://doi.org/10.1016/j.visres.2015.12.006

Wolfe, J. M., Cain, M. S., \& Aizenman, A. M. (2019). Guidance and selection history in hybrid foraging visual search. Attention, Perception, \& Psychophysics, 81(3), 637-653. https://doi.org/10.3758/s13414-018-01649-5

Wolfe, J. M., Cain, M. S., \& Alaoui-Soce, A. (2018). Hybrid value foraging: How the value of targets shapes human foraging behavior. Attention, Perception, \& Psychophysics, 80(3), 609-621. https://doi.org/10.3758/s13414-017-1471-x

Wolfe, J. M., Cave, K. R., \& Franzel, S. L. (1989). Guided search: An alternative to the feature integration model for visual search. Journal of Experimental Psychology: Human Perception and Performance, 15(3), 419-433. https://doi.org/10.1037/0096-1523.15.3.419

Wolfe, J. M., \& Horowitz, T. S. (2017). Five factors that guide attention in visual search. Nature Human Behaviour, 1(58), 1-8. https://doi.org/10.1038/s41562-017-0058

Wolfe, J. M., \& Van Wert, M. J. (2010). Varying Target Prevalence Reveals Two Dissociable Decision Criteria in Visual Search. Current Biology, 20(2), 121-124. https://doi.org/10.1016/j.cub.2009.11.066 